\title{
MicroRNAs expression profiles as biomarkers and therapeutic tools in Turkish patients with chronic myeloid leukemia
}

\author{
Yurt $\mathrm{M}^{1}$, Ayyildiz $\mathrm{O}^{2}$, Karakus $\mathrm{A}^{2}$, Nursal $\mathrm{AF}^{3}$, Isi $\mathrm{H}^{1}$ \\ Department of Medical Biology, Faculty of Medicine, Dicle University, Diyarbakir, Turkey. \\ feyda.nursal@gmail.com
}

\begin{abstract}
AIM: In $95 \%$ of Chronic myeloid leukemia (CML) patients, chromosomal translocation resulting in the formation of the Philadelphia $(\mathrm{Ph})$ chromosome $(\mathrm{t}: 9 ; 22)$ is observed, which in turn leads to the formation of the $B C R-A B L$ fusion gene. MicroRNAs (miRNAs) are a group of small and non-coding RNAs modulating gene expression via binding to the target mRNAs. We aimed to characterize the expression profiles of various miRNAs in different stages of $\mathrm{Ph}(+) \mathrm{CML}$ patients.

METHODS: This case-controlled study was conducted in $75 \mathrm{CML}$ patients and 25 healthy controls. The subjects were categorized into 4 groups; newly diagnosed patients, treatment-response patients, treatmentfailure patients, and healthy controls. Expressions of miRNAs was analyzed by RT-PCR.

RESULTS: miR-150 expression was downregulated in the treatment failure patients compared to the control group ( $p=0.003212$ ) while miRNA 148b expression up-regulated in the treatment failure patients than the control group ( $p=0.038016$ ). miR-10a expression was up-regulated in newly diagnosed and treatment response patients compared to control group $(p=0.003934, p=0.000292$, respectively). It was found that miR-10a expression increased 11.17- fold in newly diagnosed patients and 9.82-fold in treatment response patients than in the control group.

CONCLUSION: Our data suggest that expression profiles of miR-10a, miR-150, and miRNA 148b were correlated as biomarker and therapeutic tool in Turkish patients with CML (Tab. 2, Fig. 1, Ref. 30). Text in PDF www.elis.sk.

KEY WORDS: chronic myeloid leukemia, microRNAs, new diagnosis, treatment response, treatment failure.
\end{abstract}

\section{Introduction}

Chronic myeloid leukemia (CML) is a clonal hematopoietic stem cell disease, manifested by an expansion of immature granulocytes (blasts) which amass in the bone marrow and hinder the normal blood cell production (1). Epidemiologic studies report that the frequency of CML is approximately 1-2 cases/100,000 people, and most CML cases are old people (median age on diagnosis: 65 years) (2). In $95 \%$ of the cases, chromosomal translocation leading to the formation of the Philadelphia $(\mathrm{Ph})$ chromosome is seen, which in turn causes the emergence of the $B C R-A B L$ fusion gene (3). While the $B C R-A B L$ fusion impels the initial chronic phase of CML, further genomic changes that render leukemia cells resistant to Tyrosine kinase inhibitors (TKIs) therapy and independent of $B C R-A B L$ play a role in the progression of the disease.

MicroRNAs (miRNAs) are a class of small noncoding RNAs of 21-22 in length that play a role in the regulation of gene ex-

${ }^{1}$ Department of Medical Biology, Faculty of Medicine, Dicle University, Diyarbakir, Turkey, ${ }^{2}$ Department of Hematology, Faculty of Medicine, Dicle University, Diyarbakir, Turkey, and ${ }^{3}$ Department of Medical Genetics, Faculty of Medicine, Hitit University, Corum, Turkey

Address for correspondence: A.F. Nursal, Department of Medical Genetics, Faculty of Medicine, Hitit University, Corum, Turkey.

Phone: +90.364.2221100, Fax: +90.364.2221102 pression at the posttranscriptional level (4). They are evolutionarily well-conserved and, by binding to their target transcript in the 3'-UTR, can hinder the translation of proteins and destabilize the target mRNAs (5). Because of the regulation exerted by miRNAs on numerous biological processes, such as proliferation, apoptosis and differentiation, impairment of the MiRNA regulatory network may be involved in tumorigenesis. MiRNAs can serve as both oncogenes and tumor suppressors (6). miRNAs play role in CML pathogenesis: some miRNAs are up-regulated while others are down-regulated in the peripheral blood of CML patients (7). Furthermore, some studies suggest that different expression levels of miRNAs are seen in CML patients with good and poor response to TKI therapy.

Given that miRNAs have been implied to play a major role in leukemogenesis and that expression profiles of miRNAs have been linked to several cancer types, we aimed to test whether expression profiles of miR-150, miR-10a, miR-148b, and miR-130b display in patients at different stages of CML including newly diagnosed, treatment-response and therapy-failure patients.

\section{Materials and methods}

\section{Study population}

This study was conducted from April 2017 to January 2018 and included 75 (32 males and 43 females) patients with CML 


\section{9-163}

recruited from the Hematology Department of Dicle University Research and Education Hospital, Diyarbakir, Turkey. An age- and gender-matched control group composed of 25 healthy subjects (11 males and 14 females) without a medical history of cancer or other chronic diseases. The diagnosis of CML was based on standard clinical data and confirmed by molecular analyses. The current CML treatments include hydroxyurea, bone marrow transplantation or TKIs. We separated all subjects into four groups: Group 1(n $=25)$ : newly diagnosed patients, Group $2(n=25)$ : treatment response patients, Group $3(\mathrm{n}=25)$ : treatment failure patients, Group $4(\mathrm{n}=25)$ : healthy control group. Treatment response is characterized as $B C R-A B L<0.1 \%$. Therapy failure is defined here as complete hematological response with failure to achieve complete cytogenetic remission. All subjects signed a written consent form after being informed about the details of the study. Informed consent in accordance with the study protocol was approved by the Ethics Committee of the Dicle University. All the procedures were rigorously conducted following the terms of the Declaration of Helsinki.

\section{Bcr-Abl analysis}

Using RBC Lysis Solution and QiAmp RNA Blood Mini Kit kits, leukocytes from $10 \mathrm{ml}$ whole blood were obtained first, then RNA was isolated from leukocytes. (Qiagen, Germantown, MD). Then, the total RNA quality was checked using the spectrophotometric method (Maestrogen, MaestroNano Spectrophotometer, USA). Then, the cDNAs were synthesized using a Ipsogen RT Kit (Qiagen Hilden, Germany). This was used for all samples to perform reverse transcription from $1 \mu \mathrm{g}$ of total RNA in a Labcycler Thermal Cycler (SenSoquest). Following these procedures, the cDNA step was completed. Then for this step, the mix was loaded onto the Real-Time PCR (Rotor-Gene, Qiagen Hilden, Germany) using the Ipsogen BCR-ABL1 Mbcr IS-MMR Kit. The RT-PCR step was switched to look at Bcr-Abl levels. According to the results, groups with newly diagnosed, treatment-responsive and unresponsive patients were formed.

\section{RT-PCR analysis of plasma samples for miRNA levels}

miRNA isolation was performed using miRNeasy Serum/ Plasma Kit. The miRNAs obtained were stored at $-80{ }^{\circ} \mathrm{C}$ until the Real-Time PCR (RT-PCR) stage. Then, the total RNA quality was checked using the spectrophotometric method (Maestrogen, MaestroNano Spectrophotometer, USA). Then, the cDNAs were synthesized using QiagenmiScript II Reverse Transcription Kit II (Qiagen,Sabioscience). This was used for all samples to perform reverse transcription from $0,5 \mu \mathrm{g}$ of miRNA in a Labcycler Thermal Cycler (SenSoquest). For RT-qPCR analysis, diluted cDNA was used as a template). The cDNA enrichment step proceeded then. cDNAs were enriched using the miScriptPreAMP PCR Kit. RT-PCR was introduced to determine the expression levels of miRNAs. The cDNA, in combination with a miScript SYBR Green PCR kit (Qiagen, Germantown, MD), was used for RTqPCR miScriptPrimer Assays. RT-qPCR was run in a Rotor-Disc 72 with reaction volumes that used $25 \mu$ of reaction mixture for 40 cycles of $95{ }^{\circ} \mathrm{C}$ for $15 \mathrm{~min}, 94^{\circ} \mathrm{C}$ for $15 \mathrm{~s}, 55^{\circ} \mathrm{C}$ for $30 \mathrm{~s}$ in
Tab. 1. Mean ages of the subjects.

\begin{tabular}{lccccc}
\hline \multirow{2}{*}{ Groups } & \multicolumn{2}{c}{ Males } & & \multicolumn{2}{c}{ Females } \\
\cline { 2 - 3 } \cline { 5 - 6 } & $\mathrm{n}(\%)$ & Mean age & & $\mathrm{n}(\%)$ & Mean age \\
\hline Group 1 & $10(10)$ & $61.00 \pm 18.42$ & & $15(15)$ & $41.00 \pm 10.21$ \\
Group 2 & $10(10)$ & $48.60 \pm 18.57$ & & $15(15)$ & $48.87 \pm 11.54$ \\
Group 3 & $12(12)$ & $51.25 \pm 17.94$ & & $13(13)$ & $49.00 \pm 16.66$ \\
Group 4 & $11(11)$ & $53.36 \pm 12.64$ & & $14(14)$ & $47.64 \pm 16.64$
\end{tabular}

Group 1: newly diagnosed patients; Group 2: treatment response patients; Group 3: treatment failure patients; Group 4: healthy controls.

the QiagenRotorgene Q (Qiagen, Hilden). The reaction mixture contained $10 \mu \mathrm{L}$ SYBR Green Master, $2 \mu \mathrm{L}$ miscript primer assay, $2 \mu \mathrm{L}$ universal primer $1 \mu \mathrm{L}$ of DNase/RNase-free distilled water and $5 \mu \mathrm{L}$ of cDNA template. It is known that Snord61 is the most stable reference gene under exposure to pesticide, so we selected this gene in our study. Reference sequence numbers for all primers were obtained from the set shown in Table 1. (Genes were produced for Human (Homo sapiens) by miScript Primer Assays (QiagenSABiosciences USA).

$\mathrm{Ct}$ values were exported to an Excel file, a table of $\mathrm{Ct}$ values was created, and the table was uploaded to the data analysis web tool (8). The samples were classified and tagged as the control and the patient groups. Manually selected reference genes were used to normalize the obtained $\mathrm{Ct}$ values. The online tool uses the $\Delta \Delta \mathrm{Ct}$ method to calculate the fold-change/regulation. Briefly, $\Delta \mathrm{Ct}$ was first calculated between the studied gene of interest (GOI) and an average of housekeeping genes (HKG), and $\Delta \Delta \mathrm{Ct}$ was further determined by subtracting the $\Delta \mathrm{Ct}$ (control) value from the $\Delta \mathrm{Ct}$ (experiment). Fold-change was assessed using the formula: $2^{\wedge}(-\Delta \Delta \mathrm{Ct})$. The data analysis report was exported from QIAGEN web portal (8).

\section{Fold-change}

Fold-change $\left(2^{\wedge}(-\Delta \Delta \mathrm{Ct})\right)$ is the ratio of the normalized gene expression $\left(2^{\wedge}(-\Delta \mathrm{Ct})\right)$ in the test sample over the normalized gene expression $\left(2^{\wedge}(-\Delta \mathrm{Ct})\right)$ in the control sample. Fold-regulation, on the other hand, is a representation of the fold-change results in a biologically meaningful manner. In brief, fold-regulation is equal to fold-change in cases of positive- or up-regulation, where the fold-change value is greater than one. Conversely, when the fold-change is less than one, which is indicative of a negative- or down-regulation, fold-regulation equals to (-1/(fold-change)). Student's t-test was used to assess the relationship between the replicate $2^{\wedge}(-\Delta \mathrm{Ct})$ values obtained from each gene in the control group and those from the patient group, and the $\mathrm{p}$ values were calculated.

\section{Statistical analysis}

Real-time PCR data were analyzed using the Gene Globe Data Analysis Center (Qiagen). The raw data based on the $\Delta \Delta \mathrm{Ct}$ method were normalized using the Snord61 housekeeping gene. Foldchange values $\leq 2$ were considered to be up- or down-regulated. Comparison of continuous data was performed using Student's $\mathrm{t}$-test, either paired or unpaired, as needed. $\mathrm{p}$ values less than 0.05 were deemed statistically significant. 
Tab. 2. Fold change values according to groups.

\begin{tabular}{lcc}
\hline Genes & Fold-Changes & $\mathrm{p}$ \\
\hline Group 1 & & \\
Snord61 & 1.00 & Housekeeping gene \\
miR-150 & 0.98 & 0.863306 \\
miR-10a & 11.17 & 0.003934 \\
miR-148b & 3.78 & 0.125377 \\
miR-130b & 1.08 & 0.617569 \\
\hline Group 2 & & \\
Snord61 & 1.00 & Housekeeping gene \\
miR-150 & 1.58 & 0.237789 \\
miR-10a & 9.82 & 0.000292 \\
miR-148b & 1.97 & 0.451892 \\
miR-130b & 1.03 & 0271973 \\
\hline Group 3 & & \\
Snord61 & 1.00 & Housekeeping gene \\
miR-150 & 0.08 & 0.003212 \\
miR-10a & 2.85 & 0.375747 \\
miR-148b & 0.35 & 0.038016 \\
miR-130b & 0.77 & 0.105655 \\
\hline Group 4 & & \\
Snord61 & 1.00 & Housekeeping gene \\
miR-150 & 0.05 & 0.000001 \\
miR-10a & 0.29 & 0.006950 \\
miR-148b & 0.18 & 0.003557 \\
miR-130b & 0.75 & 0.439569 \\
\hline Group 1: newly diagnosed; Group 2: treatment response; Group 3: treatment failure; \\
Group 4: healthy control. & &
\end{tabular}

\section{Results}

A total of 100 subjects including 75 CML patients and 25 unrelated healthy subjects were evaluated. There were $43 \%$ females and $32 \%$ males in patients group. The mean age in the groups is shown in Table 1. To decipher a potential miRNA expression signature, we have analyzed expression profiles of miRNAs of newly diagnosed patients, treatment response patients, treatment failure patients, and compared them with healthy controls. The expression profiles of the groups are summarized in Table 2.

miR-150 expression was down-regulated in the treatment failure patients compared to the control group $(p=0.003212)$ while

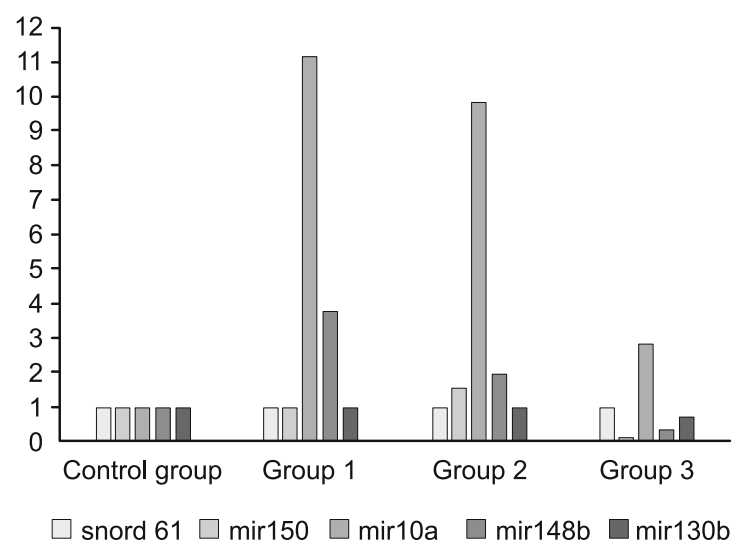

Fig. 1. Graphic view of the fold change of expression prophiles of different miRNAs in groups (Group 1: newly diagnosed; Group 2: treatment response; Group 3: treatment failure and control group).
miRNA 148 b expression was up-regulated in the treatment failure patients compared to the control group $(p=0.038016)$.

miR-10a expressions were up-regulated (fold change $>1$ ) in newly diagnosed and treatment response patients compared to healthy control group $(\mathrm{p}=0.003934, \mathrm{p}=0.000292$, respectively). It was found that miR-10a expression increased 11.17-fold in newly diagnosed patients and 9.82-fold in the treatment response patients compared to the healthy control group. Although it was not statistically significant, miR-10a expression was 2.85 fold higher in the treatment failure patients compared to the healthy control group (fold change $>1)(\mathrm{p}=0.375747)$.

miR-130b expression differences were not statistically significant in all groups according to fold changes.

Graphic view of the fold changes of expression prophiles of different miRNAs in groups is presented in Figure 1.

\section{Discussion}

CML is a group of cancers that are manifested by a unique genetic distortion, the breakpoint cluster region-Abelson murine leukemia viral oncogene homolog 1 (BCR-ABL1) fusion gene (9). Owing to the fact that this molecular lesion leads to CML, it was possible to create an effective targeted therapy that selectively hinders the tyrosine kinase activity of distorted BCR-ABL protein. Imatinib (IM) is a targeted therapy drug that can recognize and inactivate tyrosine kinase activity of the BCR-ABL oncoprotein, thus blocking leukemogenesis. Treatment of CML patients with IM produced very good responses, with regard to symptom management and hematological parameters. On the other hand, due to constant IM presence, primary and secondary resistance as well as molecular evidence of persistent malignancy have been observed in many CML patients (10). In some cases, point mutations in the BCR-ABL kinase domain have been increased in the development of IM resistance, secondary resistance particularly, eventually causing treatment failure. Resistance and intolerance to treatment remain a major clinical problem.

miRNAs are short, noncoding RNAs that are involved in posttranscriptional modulation of messenger RNAs (mRNAs). The human genome contains approximately 2000 distinct miRNAs (11). The miRNAs play crucial roles in tumorigenesis and cancer progression via regulation of neoplasia onset, growth, metastasis, and resistance to treatment (12), in agreement with the role of miRNAs in several developmental processes, their misregulation can largely promote the phenotypic features of all cancer subtypes studied so far. The most common deregulated miRNAs in CML include miR-10a, miR-17/92, miR-150, miR-203, and miR-328 (13). In CML, enhanced expression of $m i R-150$ and $m i R-146 a$, and decreased expression of miR-142-3p and miR-199b-5p were seen after 15 days of TKI therapy (14), indicating that this drug has the capacity to rearrange the miRNA profiles of tumor cells.

In this study, we analyzed 75 BCR-ABL positive patients. Our goal was to identify distinctive miRNA signatures for CML subgroups. miR-150 has invariably appeared to be down-regulated across multiple studies suggesting it as a potential candidate for early CML diagnosis. Multiple reports imply that reduced expres- 


\section{9-163}

sion of $m i R-150$ represents poor prognosis and a more severe state ofCML, whereas it was reported that reintroduction of $m i R-150$ relieved symptoms in cell lines (15). Down-regulation of miR-150 was seen in $\mathrm{CD}_{3} 4^{+}$cells derived from six chronic phase CML patient samples (15), indicating that the down-regulation of this miRNA is involved in disease onset. Moreover, evidence for down-regulation of $m i R-150$ as a diagnostic biomarker of CML was demonstrated in a study that used a RT-PCR method in 50 newly diagnosed chronic phase CML patients, and found important down-regulation of $m i R$ 150 (16). A study in 2010 conducted a microarray analysis in 10 chronic phase CML patients and validated the potency of $m i R$ 150 as a biomarker for CML diagnosis (14). miR-150 expression levels can also be helpful as a biomarker for treatment response. The down-regulation of miR-150 was found in the BCR-ABL transformed leukemia cell line Mo7e-p210 (megakaryoblast), and could be reestablished in response to IM treatment (15). In this study, we found that miR-150 expression was down-regulated in patients with treatment failure compared to healthy controls $(\mathrm{p}=0.003212)$. Our results were compatible with those found in other studies.

There is growing evidence suggesting that $\mathrm{miR}-10 \mathrm{a} / \mathrm{b}$ may serve as new oncogene in several types of human cancer, such as metastatic breast cancer, pancreatic cancer, esophageal cancer, hepatocellular carcinoma, nasopharyngeal carcinoma and colorectal cancer (17). These findings advocate that miR-10a and miR-10b are vigorously expressed in highly metastatic cancer and play significant roles in metastasis. $m i R-10 a$ is a potential candidate for CML diagnosis. Using a Q-RT PCR method in 85 newly diagnosed chronic phase CML patients, down-regulation of $m i R-10 a$ was seen in $71 \%$ of patients, exhibiting its clinical pertinence as a biomarker for diagnosis (15). Other studies have also investigated the pertinence of $\mathrm{miR}-10 \mathrm{a}$ as a biomarker for drug response. miR-10alevels in the Mo7e-p210 cell line were unaffected by IM treatment (14), nevertheless, a significant increment of $m i R-10 a$ was seen in microarray analysis of patient samples 15 days after IM treatment (14). In the present study, we found that miR-10a expression was up-regulated 11.17- fold in newly diagnosed and 9.82-fold in treatment response patient groups ( $p$ $=0.003934, \mathrm{p}=0.000292$, respectively) (Tab. 2). It was thought that miR-10a expression was associated with treatment response.

miR-148a, miR-148b, and miR-152 are three members of the miR-148/152 family. They have similar sequences, configuration, and the same seed region (18). All of them have been reported to be down-regulated/up-regulated in numerous distinct types of tumors (e.g., gastrointestinal, ovarian, hepatocellular carcinoma, and pancreatic cancer) (19). Some studies have shown that members of the miR-148/152 family are expressed differentially in hematological malignancies including acute lymphoblastic leukemia (ALL), multiple myeloma (MM), and lymphoma (20-23). Huang et al. found that six miRNAs such as miR-148a were significantly up-regulated in the plasma of MM patients and high levels of miR-148a were associated with shorter relapse-free survival times (22). Wang et al. found a significantly lower expression of down-regulated miR-148/152 in AML patients compared to healthy controls (24). It was reported that low expression of miR-148b was found in a subset of CML patients with stable complete molecular responses after stopping IM treatment (25). In this study, it was detected that miR-148b expression was up-regulated in treatment- failure patient group compared to the control group $(\mathrm{p}=0.038016)$.

miR-130a plays distinct roles as oncogene or tumor suppressor gene to mediate diverse biological processes by modulating several canonical pathways or target genes in various cancer types. It has been found that the expression of miR-130a is distorted in some types of cancer, overexpressed in adult T cell leukemia (ATL) (26), gastric cancer (27), but down-expressed in bladder cancer (28), and chronic lymphocytic leukemia (29). Ding et al identified miR-130a as significantly over-expressed in t $8 ; 21)$ AML(30). We found miR-130b expression showed no significant differences in all groups $(\mathrm{p}>0.05)$.

\section{Conclusion}

In summary, our data demonstrated that miR-150, miR-10a, and miR-148b are deregulated at different stages of CML. The results of this study summarize the mechanisms by which miRNAs may play a role in CML pathogenesis. However, if they act in BCR-ABL dependent or independent manner has to be further investigated.

\section{References}

1. Jurkovicova D, Lukackova R, Magyerkova M et al. microRNA expression profiling as supportive diagnostic and therapy prediction tool in chronic myeloid leukemia.Neoplasma 2015; 62 (6): 949-958.

2. Hehlmann R, Hochhaus A, Baccarani M. European LeukemiaNet: Chronic myeloid leukaemia. Lancet 2007; 370 (9584): 342-350.

3. Kalidas M, Kantarjian H, Talpaz M. Chronic myelogenous leukemia. JAMA 2001; 286 (8): 895-898.

4. Lagos-Quintana M, Rauhut R, Lendeckel W, Tuschl T. Identification of novel genes coding for small expressed RNAs. Science 2001; 294 (5543): 853-858.

5. Baek D, Villen J, Shin C, Camargo FD, Gygi SP, Bartel DP. The impact of microRNAs on protein output. Nature 2008; 455 (7209): 64-71.

6. Garzon R, Calin GA, Croce CM. MicroRNAs in cancer. Annu Rev Med 2009; 60:167-179.

7. Zhao H, Wang D, Du W, Gu D, Yang R. MicroRNA and leukemia: tiny molecule, great function. Crit Rev Oncol Hematol 2010; 74 (3): 149-155.

\section{8. http://www.qiagen.com/geneglobe.}

9. Sun QC, Liu MB, Shen HJ et al. Inhibition by imatinib of expression of O-glycan-related glycosyltransferases and tumor-associated carbohydrate antigens in the K562 human leukemia cell line. Asian Pac J Cancer Prev 2013; 14 (4): 2447-2451.

10. Jiye A, Qian S , Wang G et al. Chronic Myeloid Leukemia Patients Sensitive and Resistant to Imatinib Treatment Show Different Metabolic Responses.PLoS One 2010; 5 (10): e13186.,

11. Friedländer MR, Lizano E, Houben AJ et al. Evidence for the biogenesis of more than 1, 000 novel human microRNAs. Genome Biol 2014; 15 (4): R57.

12. Xue J, Niu J, Wu J, Wu ZH. MicroRNAs in cancer therapeutic response: friend and foe. World J Clin Oncol 2014; 5 (4): 730-743. 
13. Gordon JE, Wong JJ, Rasko JE. MicroRNAs in myeloid malignancies. Br J Haematol 2013; 162 (2): 162-176.

14. Flamant S, Ritchie W, Guilhot J et al. Micro-RNA response to imatinib mesylate in patients with chronic myeloid leukemia. Haematologica 2010; 95 (8): 1325-1333.

15. Agirre X, Jiménez-Velasco A, San José-Enériz E et al. Down-regulation of hsa-miR-10a in chronic myeloid leukemia CD34+ cells increases USF2-mediated cell growth. Mol Cancer Res 2008; 6 (12): 1830-1840.

16. Fallah P, Amirizadeh N, Poopak B et al. Expression pattern of key microRNAs in patients with newly diagnosed chronic myeloid leukemia in chronic phase. Int J Lab Hematol 2015; 37 (4): 560-568.

17. Bi L, Sun L, Jin Z, Zhang S, Shen Z. MicroRNA-10a/b are regulators of myeloid differentiation and acute myeloid leukemia. Oncol Lett 2018; 15 (4): 5611-5619.

18. Calin GA, Sevignani C, Dumitru CD et al. Human microRNA genes are frequently located at fragile sites and genomic regions involved in cancers. Proc Natl Acad Sci USA 2004; 101 (9): 2999-3004.

19. Chen Y, Song YX, Wang ZN. The MicroRNA-148/152 Family: Multifaceted Players. Mol Cancer 2013; 12: 43.

20. Rio-Machin A, Ferreira BI, Henry T et al. Downregulation of specific miRNAs in hyperdiploid multiple myeloma mimics the oncogenic effect of IgH translocations occurring in the non-hyperdiploid subtype. Leukemia 2013; 27 (4): 925-931.

21. Wu Y, Liu GL, Liu SH, Wang CX, Xu YL, Mao P. MicroRNA-148b enhances the radiosensitivity of non-Hodgkin's Lymphoma cells by promoting radiation-induced apoptosis. J Radiat Res 2012; 53 (4): 516-525.

22. Huang JJ, Yu J, Li JY, RQ Z. Circulating microRNA expression is associated with genetic subtype and survival of multiple myeloma. Med Oncol 2012; 29 (4): 2402-2408
23. Stumpel DJ, Schotte D, Lange-Turenhout EA et al. Hypermethylation of specific microRNA genes in MLL-rearranged infant acute lymphoblastic leukemia: Major matters at a micro scale. Leukemia 2011; 25 (3): 429-439.

24. Wang XX, Zhang R, Li Y. Expression of the miR-148/152 Family in Acute Myeloid Leukemia and its Clinical Significance. Med Sci Monit 2017; 23: 4768-4778.

25. Ohyashiki JH, Ohtsuki K, Mizoguchi I et al. Downregulated microRNA-148b in circulating PBMCs in chronic myeloid leukemia patients with undetectable minimal residual disease: a possible biomarker to discontinue imatinib safely. Drug Des Devel Ther 2014; 8: 1151-1159.

26. Ishihara K, Sasaki $\mathbf{D}$, Tsuruda $\mathbf{K}$ et al. Impact of miR-155 and miR-126 as novel biomarkers on the assessment of disease progression and prognosis in adult T-cell leukemia. Cancer Epidemiol 2012; 36 (6): $560-565$.

27. Jiang H, Yu WW, Wang LL, Peng Y. MiR-130a acts as a potential diagnostic biomarker and promotes gastric cancer migration, invasion and proliferation by targeting RUNX3. Oncol Rep 2015; 34 (3): $1153-1161$.

28. Ratert N, Meyer HA, Jung M. miRNA profiling identifies candidate mirnas for bladder cancer diagnosis and clinical outcome. J Mol Diagn 2013; 15 (5): 695-705.

29. Kovaleva V, Mora R, Park YJ et al. miRNA-130a targets ATG2B and DICER1 to inhibit autophagy and trigger killing of chronic lymphocytic leukemia cells. Cancer Res 2012; 72 (7): 1763-1772.

30. Ding C, Chen SN, Macleod RAF et al. MiR-130a is aberrantly overexpressed in adult acute myeloid leukemia with $\mathrm{t}(8 ; 21)$ and its suppression induces AML cell death. Ups J Med Sci 2018; 123 (1): 19-27.

Received June 2, 2019-. Accepted October 14, 2019. 